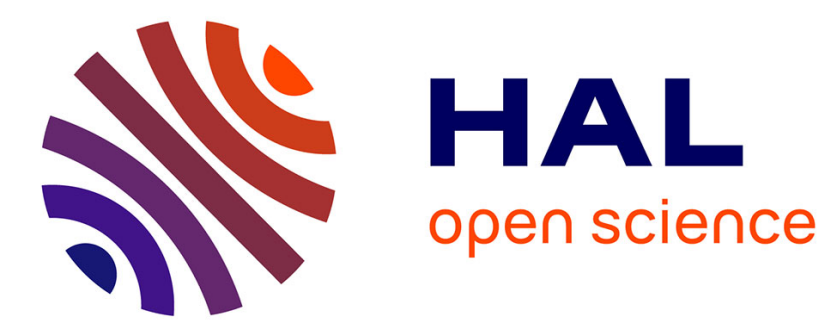

\title{
Towards a New Approach for Building Trust and Confidence in Machine Learning Data and Models: Based on an Integration of Artificial Intelligence and Blockchain (Part 3) \\ Hammou Fadili
}

\section{To cite this version:}

Hammou Fadili. Towards a New Approach for Building Trust and Confidence in Machine Learning Data and Models: Based on an Integration of Artificial Intelligence and Blockchain (Part 3). Journal of Applied Business and Economics, In press, 22 (11), 10.33423/jabe.v22i11 . hal-03093020

\section{HAL Id: hal-03093020 \\ https://hal-cnam.archives-ouvertes.fr/hal-03093020}

Submitted on 1 Feb 2021

HAL is a multi-disciplinary open access archive for the deposit and dissemination of scientific research documents, whether they are published or not. The documents may come from teaching and research institutions in France or abroad, or from public or private research centers.
L'archive ouverte pluridisciplinaire HAL, est destinée au dépôt et à la diffusion de documents scientifiques de niveau recherche, publiés ou non, émanant des établissements d'enseignement et de recherche français ou étrangers, des laboratoires publics ou privés. 


\title{
Towards a New Approach for Building Trust and Confidence in Machine Learning Data and Models: Based on an Integration of Artificial Intelligence and Blockchain (Part: 3)
}

\author{
Hammou Fadili \\ Laboratoire CEDRIC of CNAM \\ Fondation Maison Sciences de l'Homme
}

\begin{abstract}
Sentiments and opinions trends analysis and study are two important disciplines of Big Data exploitation. In our case related to Business Intelligence (BI), they make it possible to detect and extract relevant information from raw unstructured textual data, which we call: Textual Business Intelligence (TBI). It is in this context that we propose a new approach allowing to build trust and confidence in machine learning data and therefore in models: based on an integration and a convergence of artificial intelligence and Blockchain. This third part of the article will be dedicated to explaining our general process and to the experimentations.
\end{abstract}

Keywords: artificial intelligence, blockchain, machine learning, neural models, sentiments analysis, text mining

\section{INTRODUCTION}

The current project is part of the natural evolution of the researches carried out in our main research domain in its general strategy on Business Intelligence (BI), and in its orientation relative to the exploitation of Big Data. In this context, data comes mainly from users/customers sentiments and opinions, expressed via social medias, forums, blogs, sales sites, etc., on all kinds of subjects such as events, products, attitudes, etc., to express their feelings and experiences.

Analysing sentiments and experiences of users is a major problematic for companies. Several studies and statistics confirm that more than $80 \%$ of Internet users consult the comments and opinions of former users before making their own purchases. Recommendations influence our opinions on products, services, etc. and therefore influence our purchases. Companies consequently have a great interest in understanding their customers feedback and comments. They must invest massively in intelligent systems allowing automatic sentiments analysis and opinions mining, in order to be helped to adapt to users' requirements and improve their profits; in the new open world of business and economic intelligence which is the web. Despite advances in this area, several recent studies show that only $29 \%$ of companies use data in their decision. This is due to several problems, mainly related to the nature of the existing solutions which are not yet mature enough, and to their costs, which are still too expensive to implement.

The phenomena of business data analysis in the context of "Big Data", for intelligence purposes, concerned mainly the static aspects of textual data models. 
However, due to their nature in this context, data as well as their characteristics are "naturally" dynamic and therefore their handling is necessary. The analysis of dynamic aspects of data such as the study \& extraction of indicators and the analysis \& prediction of their behaviour over time, has become a major issue encouraging the emergence of new research projects, moreover difficult and complex. This complexity can be amplified if data additionally includes specific information such as anomalies, fake information, etc. In fact, the latter, which can alter the judgment and the perception that we can have on the real facts, must be considered in the treatment processes. Detecting and managing these elements is an important task for the dynamic data analysis and for the impact on the associated target indicators trends.

It is in this context that we carried out the current research project, on the dynamic data analysis, entitled:

"Designing a new approach to build trust and confidence in machine learning data and models: based on an integration and the convergence of artificial intelligence and Blockchain"

In this project, we are interested in the issues of detecting anomalies $\&$ false information and their causes in the dynamic sentiments influencing the trends prediction in associated time series/sequences, by proposing a solution, which can limit them, based on a security, certification, etc. data system, via Blockchain.

The main objectives of the project can be recalled as follows:

- Minimize the role and the intervention of a human user, in the context of big data; where processing by a human being is almost impossible to perform (large amount of data). The system should be able to "extrapolate" by exploiting a minimum of annotated \& classified (pretreated) data and learn by it itself the latent science contained in the text, in order to extract all the useful information for decision-making.

- Design and develop approaches considering the dynamic aspects of data.

- Design and develop approaches to detect anomalies and fake information in data.

- Model the prediction of targeted indicators behaviour considering anomalies and fake information.

- Study a solution that can prevent fake information, build confidence and trust in data and make reliable analysis and predictions.

\section{GENERAL PROCESS}

\section{At Blockchain Level}

This section presents the technological integration process implementing sentiments prediction by exploiting Blockchain technology. The process considers the following elements:

- At an instant $\mathrm{t}$, we can predict sentiments for the future Blocks created between $\left[t\right.$ and $\left.t+p_{2}\right]$ from the past blocks created between $\left[t\right.$ and $\left.t-p_{I}\right]$. These are parameters that can be set at the beginning.

- In the previous paragraphs, we explained the Blockchain integration for sentiments \& opinions analysis and prediction. This by storing metadata including references to documents in Blockchain. So, depending on the behavior (of a user, indicator, etc.) that we want to analyze and/or predict, we have a layer at each block level allowing to filter the desired data. The elements obtained are increased by the polarity (correctable) of the current block to enrich the features.

- After that, we train an LSTM to predict sentiments of the next block. The learning and the correction are done by comparison with the effective polarity calculated from the same next block. Of course, this is true for the blocks of the past relating to the chosen time interval $\left[t-p_{l}\right.$, $t]$ in the training phase. 
- At this step, we obtain a sequence of sequences which we submit to a hierarchical LSTM model (to set up) to predict the sequence corresponding to the predictions of the future blocks of the chosen time interval in the beginning $\left[t, t+p_{2}\right]$.

\section{FIGURE 11 \\ THE GENERAL BLOCKCHAIN LEVEL PROCESSING PROCESS}

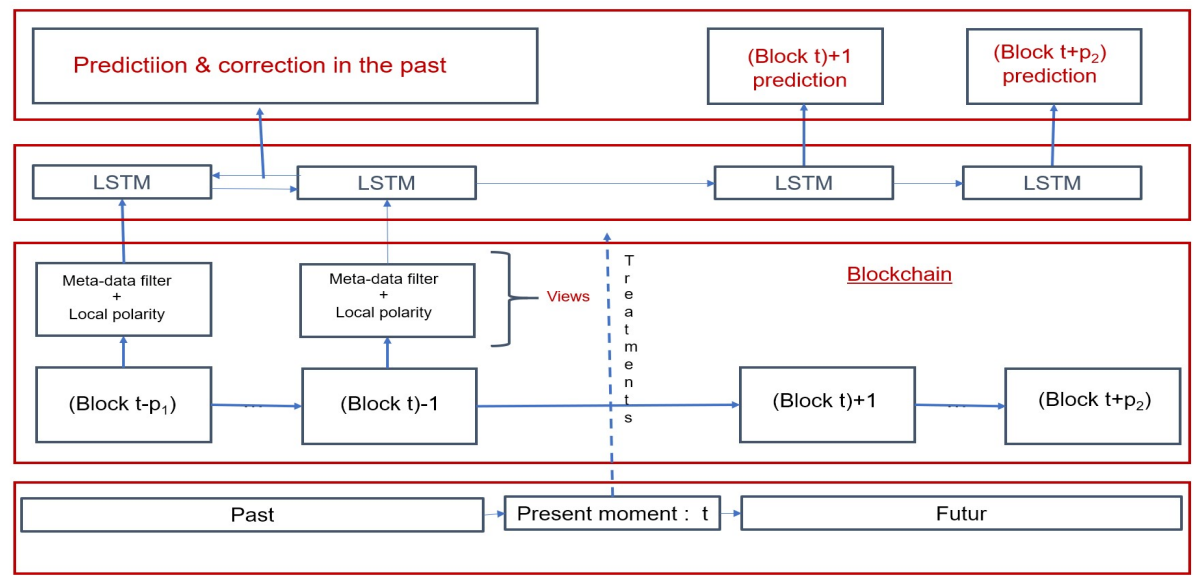

\section{At the Block Level}

This section will be devoted to the presentation of the implemented process at the processing level inside each block. As explained above, it is based on a hybrid methodology, which can combine approaches allowing the exploitation of annotated corpora (as learning dataset) and approaches based on sentiments lexicons (in this case SentiWordNet). The process consists in extracting the necessary elements from the data model and the language model for sentiments prediction, in order to feed a deep learning system for the analysis and interpretation of words meanings in the text, in the first phases of processing, followed by the calculation of the subjectivity conditioning the calculation of the polarity at an instant $t$ for the prediction of the polarity at the instant $t+1$, in the following phases.

\section{FIGURE 12}

\section{THE GENERAL BLOCK LEVEL PROCESSING PROCESS}

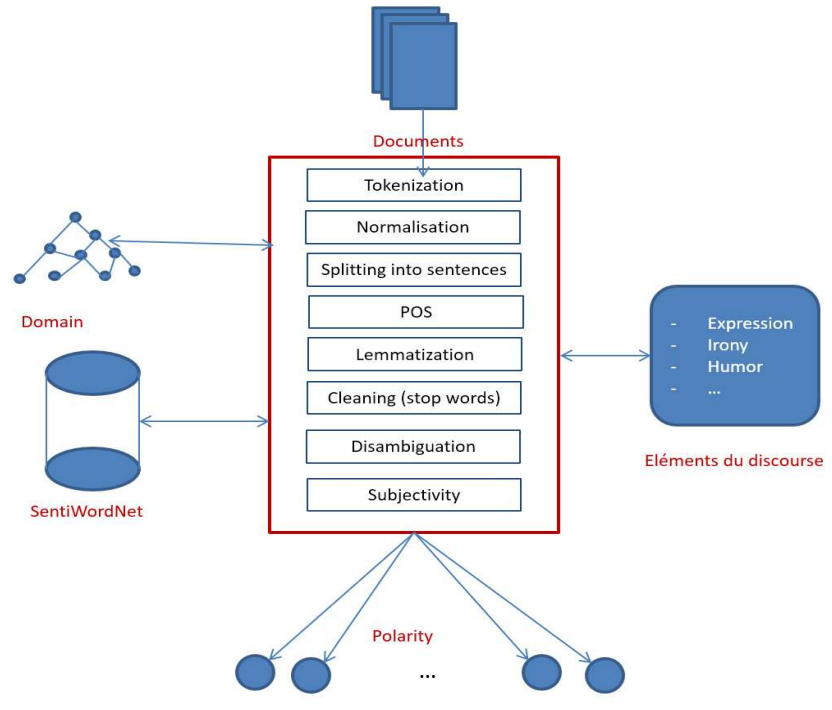




\section{Learning and Instance Model}

The learning model is obtained by unfolding the language model and the extended semantic model for sentiments prediction, described above, and by projecting the initial vector representations of words, relating to a large space (vocabulary size), in a reduced dimensions semantic space using Word2vec technology $(\mathrm{w} 2 \mathrm{v})$. The goal is to create an instances model enriched and adapted to the context of sentiments prediction; with a vector representation of reasonable size, essential for optimizing calculations.

\section{FIGURE 13 INSTANCES MODEL}

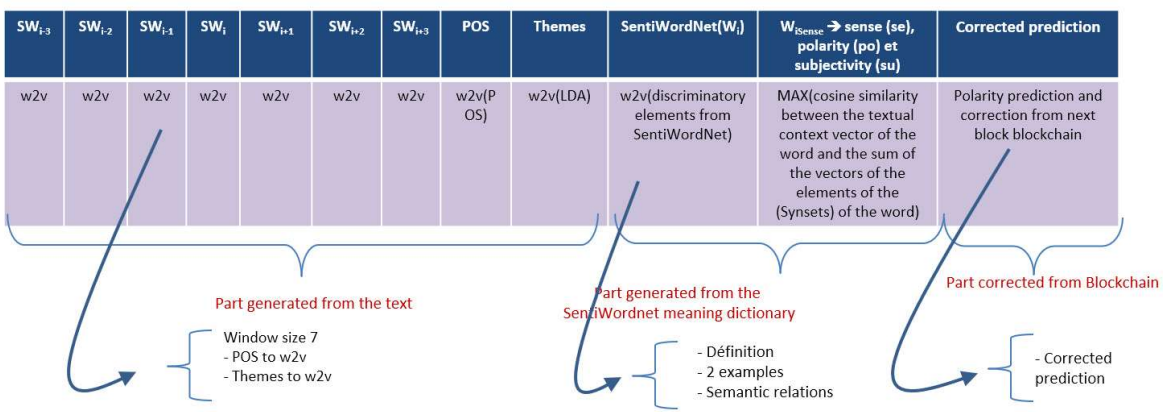

In the case of attributes composed of several words, the simplification of vectors is done by calculating the average of the vectors of each meaning.

These models are still incomplete. Our architecture being modular thanks to the flexibility of LSTMs, can allow flexible management of parameters (Features) that can be added, deleted, modified on demand.

\section{Technological Elements for Instantiating Instances Model}

The instantiation of the semantic model for machine learning takes place in several steps. The first parts concern disambiguation will not be described in this document. For more information on these parts (see Hammou Fadili 2019). The following parts concern the preparation of the instances and the calculations of subjectivities and polarities, globally exploit the results of the first phases to:

- Generate for each document an unambiguous version where each word is replaced by its true meaning in the context.

- Impact these changes at the instance level to obtain unambiguous instances.

- Enrich instances with specific parameters to sentiments analysis at time $t$.

- Increase the model by a corrected prediction parameter (calculated from the next block) at time $t+1$

- Carry out training, evaluation and testing on the instances model.

It is an important contribution, to the existing "Word Embeddings" models in the literature for natural language by disambiguating and contextualizing them (the vector of a word is obtained by the average of the vectors of its semantic context).

On another aspect related to the calculation and the prediction of polarity, the existing studied methods are interesting, but remain limited $\&$ incomplete in our opinion. They proceed by calculating the polarities of all words without exception, even for objective (non-subjective) ones. Also, existing systems do not use intermediate predictions for global prediction. To remedy this problem, on the one hand, we proceed differently: instead of training the system on all meanings, we train it only on the subjective and relevant (attention) words meanings influencing the global polarity relative to a domain (point of view). On the other hand, we consider the intermediate predictions corrected using Blockchain in the global prediction. This is a significant improvement that is not supported in any of the studied existing approaches. 


\section{System Architecture}

The general architecture consists of several main stages:

- Data preparation. We proceed to:

- Noise cancellation

- Standardization (correspondence in the vocabulary of acronyms, SMS, ontologies, etc.)

- Natural language processing (Sentence Splitter, Tokenization, Lemmatization, POS, Stop Words removal)

- Disambiguation

- Specific treatments for natural language. We treat differently:

- Expressions

- Metaphors

$\circ$ Irony

O $\ldots$

- Sentiments lexicon. We use:

- SentiWordNet

- Corpus representation. We use a vector space, generated from:

- Adequate dataset

- Machine learning. We apply algorithms for the deduction of:

○ Subjectivity

○ Polarity.

- Intermediate predictions. We apply algorithms to predict the polarity of each element:

- Intermediate prediction.

- Correction of intermediate predictions. We apply algorithms to correct the prediction of the polarity of each element from the real polarities calculated from the next block:

- Prediction correction made possible thanks to blockchain.

- Global prediction. We apply algorithms for the deduction of:

- Global prediction for future blocks of the chosen time interval at the beginning.

These elements of the architecture will be detailed in the following.

\section{The First Step}

Being in the context of the Web where the data is noisy, marked up, etc., the first phase consists first in cleaning data, before processing and generating inputs according to our data model. Next comes standardization, a very important step in data preparation. It consists of "Mappings" between elements outside of the used language vocabulary and elements of the vocabulary. This is in order to attribute a meaning to elements such as acronyms, SMS, shortcuts, abbreviations, etc. and to allow their recognition by natural language processes. This is a very significant improvement on the proposed approach. After that, we use technologies such as POS, Tokenization, Stemming and Splitting to generate the grammatical category, the local context and the context of the text (themes or topics) using latent semantic management technology LDA (Latent Dirichlet Allocation). The goal is to create one vector per word, enriched by discriminating properties of meaning (disambiguation).

Still in the data preparation phase, one of the challenges of new sentiments analysis approaches in the context of BI is to detect implicit semantics conveyed in the text. We are interested in certain specificities of natural language such as expressions / metaphors. As a first approach, we proposed to detect them, isolate them and exclude them from natural language processing processes to reserve them specific treatments. This TALN issue is a very difficult research domain that we plan to deepen in our future work.

\section{The Second Step}

It consists in using sentiments lexicons such as SentiWordNet to extract the subjectivity of the studied elements conditioning their polarities. This completes and enriches the features vector obtained from the 
previous step. As described in the general process, the association of sentiments and polarities is considered after the disambiguation and the deduction of subjectivities. This makes it possible to associate the sentiments and the polarities with senses and not with words. This is another improvement made in the project, compared to the classic processes of sentiments analysis.

\section{The Third Step}

As another innovation, we exploited the technology of "Web Embeddings" and its implementation "Word2vec" to represent words by reduced dimensions vectors. For this, we have chosen and exploited specific corpora as the basis for generating the vector space; and projected there the instances vectors (generated in the previous steps). Several corpora exist in free access; but in our approach, the choice of corpus was very important and motivated. We have exploited corpora dealing with themes close to our domain; because once projected into the learned vector space, the associated vectors with the words become relevant and close to our reality. They are based on past uses of the same words from the same domain. We were able to show that this choice obviously has a positive impact on the performance of our system and represents a considerable improvement.

\section{The Fourth Step}

Use of deep learning technology for sentiment analysis. Several studies have shown that the use of solutions based on deep learning is very interesting and even essential for sentiments analysis. It allows very abstract and complex representations of semantics, which is not supported by conventional solutions. Therefore, we have privileged and exploited them for the current project. We have also improved the complex versions of deep learning architectures such as recurrent architectures RNN and LSTM (see above).

\section{The Fifth Step}

Train the system on the generated datasets. The first dataset concerns training on data from the past, relating to chosen blockchain blocks.

\section{The Sixth Step}

The second dataset concerns the predictions at each block level. We apply algorithms to predict the polarity of each element: Intermediate prediction.

\section{The Seventh Step}

Correction of the intermediate predictions of each block. We apply algorithms to correct the prediction of the polarity of each block from the real polarities obtained from the next block: Prediction correction made possible thanks to Blockchain.

\section{The Eighth Step}

Polarity prediction of future blocks of the selected time interval. We apply algorithms for the deduction of the global prediction.

\section{The Ninth Step}

Evaluate the system on new datasets. The evaluation of the approach was carried out according to several parameters. We've tested:

- The contribution of parameters (Features) we introduced in the language, data and sentiments modeling for machine learning.

- The performance of the different classification technologies based on machine learning. This is to make the choice of the best algorithm, specifically adapted for our use case.

- The contribution of Blockchain on at least two axes: data quality and correction of intermediate predictions. 
- The impact of each type of corpus on the analysis results. For this, we projected our data into vector spaces learned from data from different themes, then compared the results for each theme. The analysis results allowed us to conclude that the closer the domain (theme), the better the results (cf. precision and loss of the system).

FIGURE 14

GENERAL ARCHITECTURE

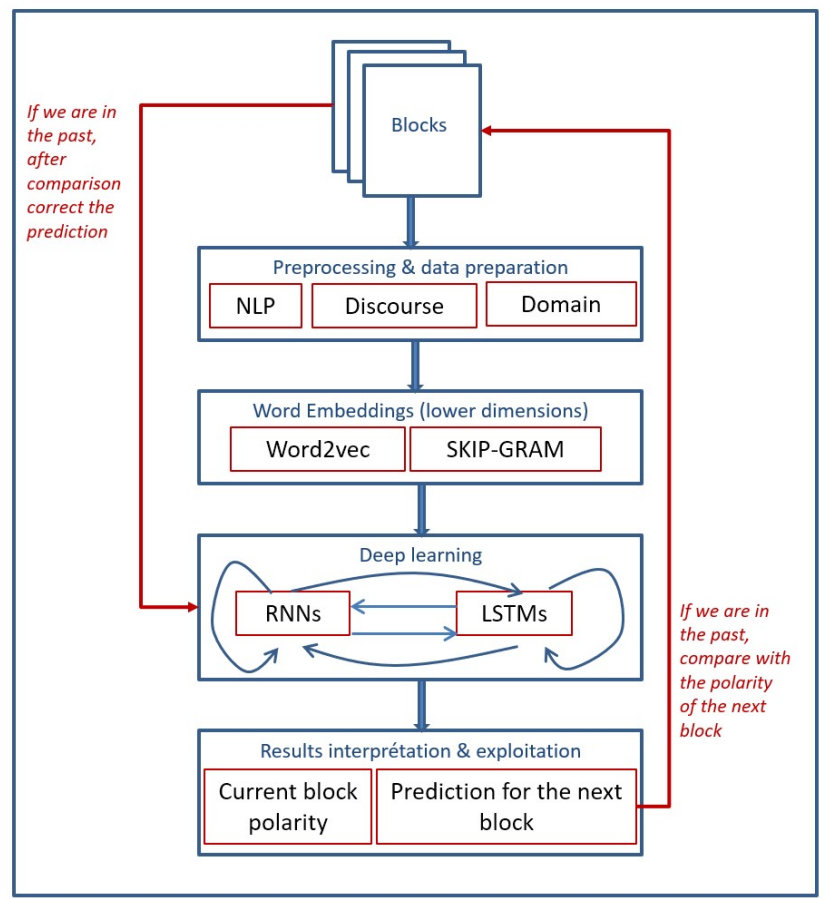

\section{TESTS AND EVALUATIONS}

\section{Test Scenarios}

The studied scenarios are as follows:

- Simulation of the exploitation of a Blockchain for automatic dynamic data management: blocks

- Automatic extraction of different semantic characteristics of words and texts for the dynamic sentiments analysis.

- Implementation of a deep learning system based on an RNN and LSTM with attention \& domain mechanisms, enriched to consider the partial predictions of blocks and their corrections from the next blocks for the past blocks in order to predict the polarity of future blocks, relative to the chosen time interval.

For a given time interval, we developed and used modules for the extraction of contextual characteristics. The workflow was implemented in Orange Canvas platform. Given that the tested tools namely Weka, RapidMiner and Orange do not support all the needs of our approach, we were obliged to develop our own deep neural network of LSTM type endowed with all the mechanisms mentioned above, for the machine learning part. This implementation runs on two levels:

- At the level of each block to calculate the current polarity,

- Then between the blocks to predict and correct the predicted polarities of each block.

Regarding the data, we generated two datasets: 
- The first to train and test the system at block level. The goal is that from the data of a block, we can calculate its polarity.

- The second was obtained from the first, by shifting the polarities of the blocks by one unit of time. The goal is to associate the polarity of the next block with the prediction of the current block. This is an important information for the instances model increasing the accuracy of the system.

The simulations took place on these two datasets in two stages. As required in "honest" machine learning systems, for each simulation we have separated the generated learning data into three parts:

- The first part for validation on $20 \%$ of the dataset, in order to optimize the hyperparameters of the system: the learning step, the type of the activation function and the number of layers.

- The rest of the dataset is divided into two parts:

- $60 \%$ for training, this in order to estimate the best coefficients $\left(w_{i}\right)$ of the neural network function, minimizing the error between the actual outputs and the desired outputs.

- $20 \%$ for testing, in order to assess the performance of the system.

For a given time interval, during all the learning phases, the system is autonomous, it is capable of generating the characteristics of the text (Features) for training, then deducing the sense of each word from the text, as well as the subjectivity and polarity of the elements on which the study relates, in this case the block as well as the step block.

\section{An Overview of First Results}

To read the following graphics:

- The red curve corresponds to the calculation of the current polarities

- The blue curve corresponds to the prediction of the next polarities

FIGURE 15

CALCULATING ACCURACY OF CURRENT POLARITIES

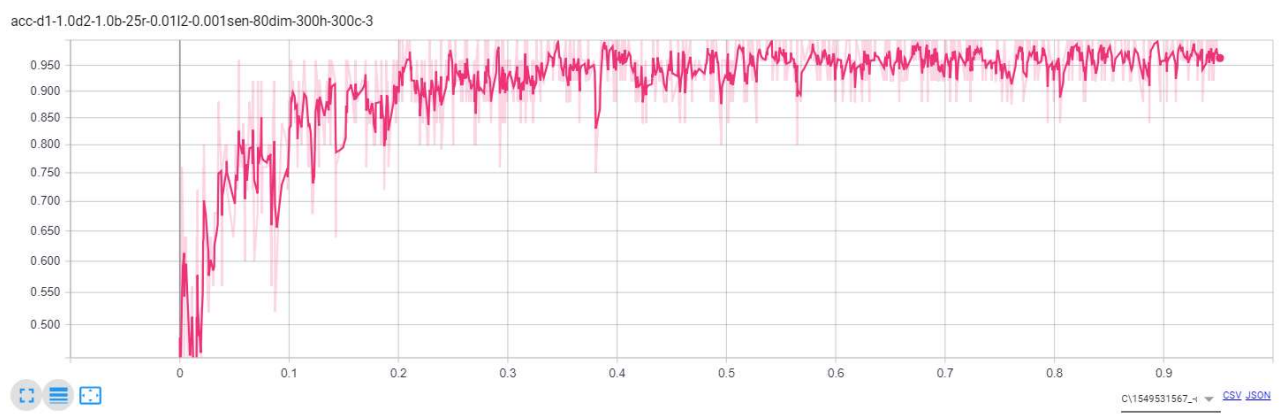

FIGURE 16 CALCULATION LOSS OF CURRENT POLARITIES

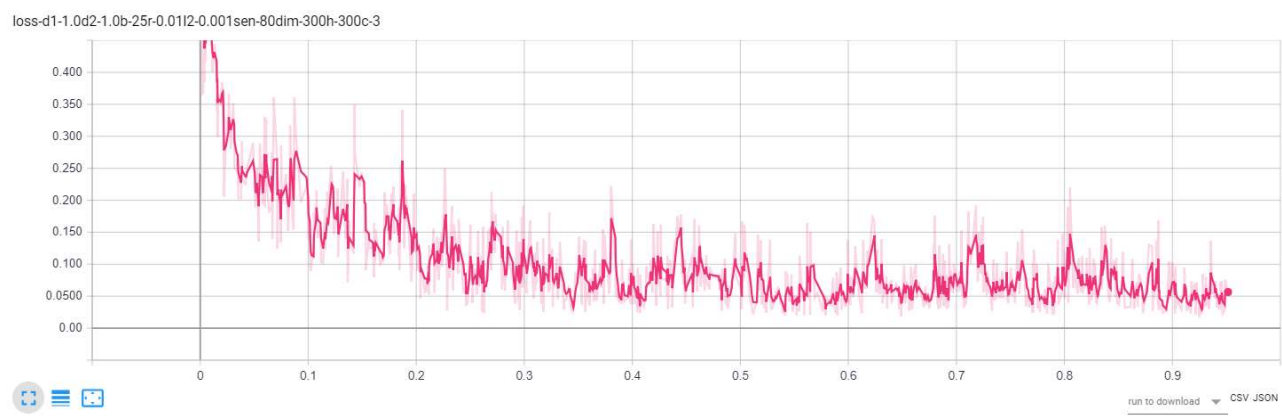


FIGURE 17

PREDICTIONS ACCURACY

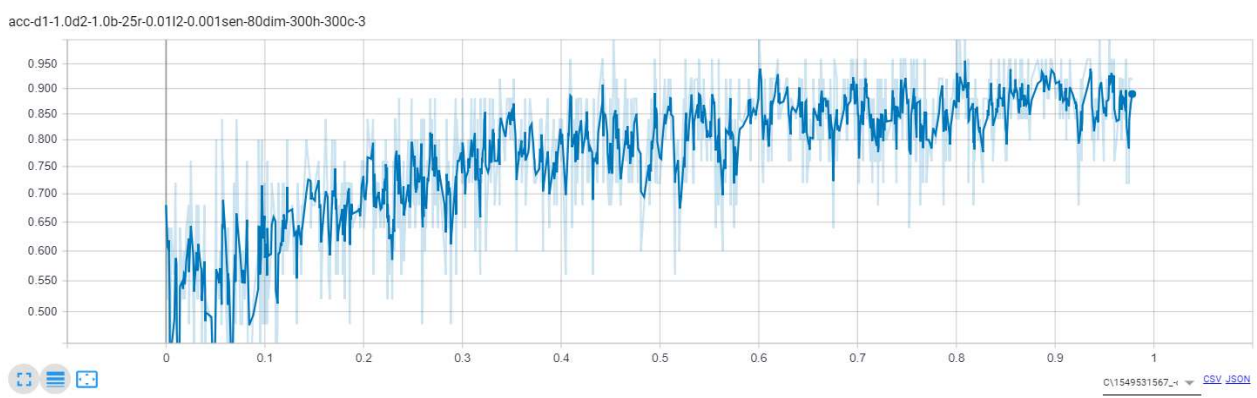

FIGURE 18

PREDICTIONS LOSS

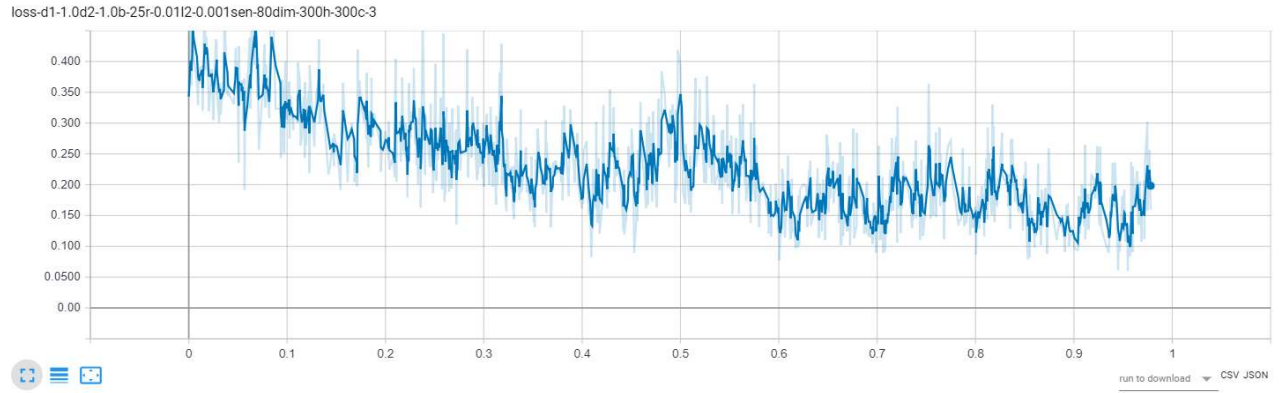

FIGURE 19

COMPARISON OF POLARITY ACCURACY AND PREDICTION

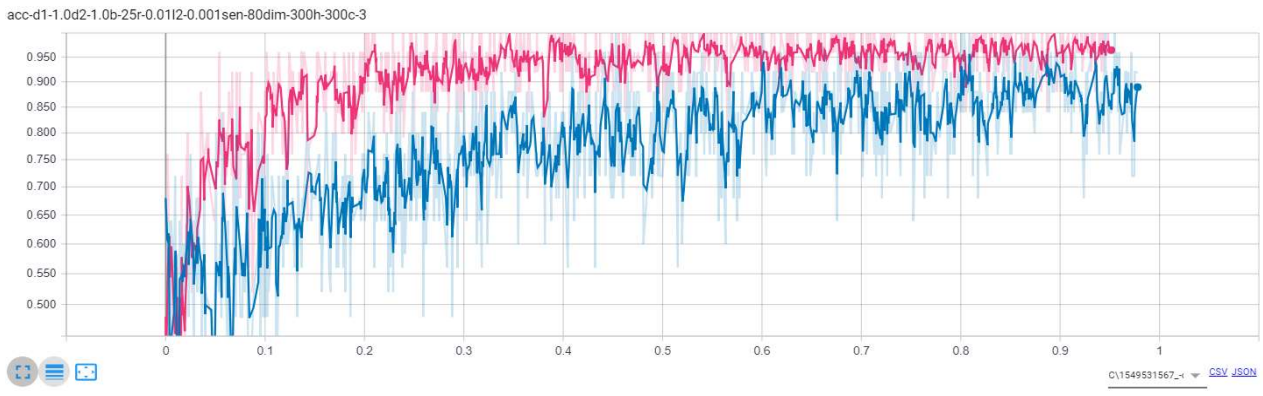

FIGURE 20

COMPARISON OF POLARITY LOSSES AND PREDICTION

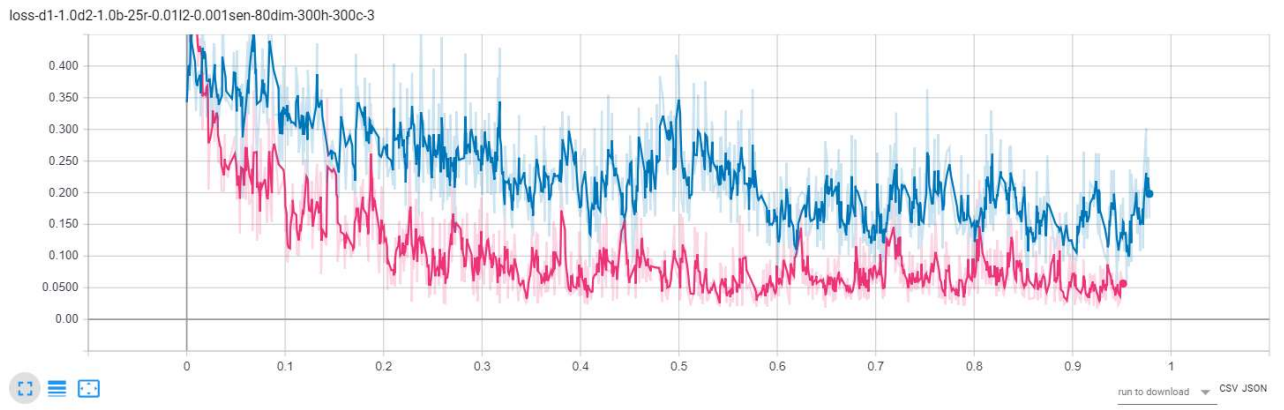




\section{Elements of Results Interpretation}

We can already notice on the graphs that the accuracy of the current polarities is better than that of the prediction: this is normal, the result of an observation is more precise than the prediction of its behaviour.

The first interesting result in these tests, however, is that the prediction trend is about the same as that of the polarity (observation). These two trends are growing and tend respectively towards interesting proportions. In our case, we can say, on the one hand, that the more the system learns, the better its performance. On the other hand, we can also say that the contribution of elementary predictions (which correspond to observations in the past) is also interesting. This is since we increased our instance model by a "true" value which is the corrected prediction provided by the Blockchain. The system combining the improvements in sentiments analysis that we have implemented and the specific improvements enriching the prediction model, provided very satisfactory results.

These same remarks are automatically valid for the loss.

\section{CONCLUSION \& PERSPECTIVES}

Sentiment analysis has become a very important discipline in the exploitation of big data. Although there are several proposals and approaches in the literature, there is today no solution which can consider all the requirements of life cycles of sentiments prediction as well as the associated treatments.

It is in this context that we proposed a solution improving the dynamic aspects of the following elements:

- The disambiguation at the beginning of the process

- The context to deduce the global semantics and thereafter the subjectivity and the polarity

- The possibility of ontology integration and other knowledge bases to solve problems such as: acronyms, text messages, expressions (expressions), etc.

- The implementation of an improved LSTM

- Support of intermediate predictions to improve overall prediction

- Learning and correcting predictions thanks to Blockchain

- Guaranteeing the data quality and predictions thanks to Blockchain

$-\quad \ldots$

Following various tests, we can conclude: the more properties available characterizing the global context for the instances, the more reliable the system is in its predictions. The exhaustive definition and the extraction of the Characteristics being complex cannot be done without difficulties, several limitations were encountered, and several were abandoned as explained previously. As a reminder, we have:

- Used and abandoned solutions based on descriptions such as ontologies; because they are difficult to set up. In fact, we have been talking about ontologies for several years, but today there are no complete ontologies, except in very limited fields. Therefore, we redirected our work towards unsupervised or mixed solutions for instantiating our extended models.

- Used and abandoned technologies based on "sparse" vector representations; because they cause combinatorial explosions in treatments. We have adopted the "word embeddings" model: new "revolutionary" representations that can be learned from corpus and projected into "semantic" reduced dimensions vector spaces (around 300). This technology was also abandoned in its initial version, because the representations are ambiguous. Therefore, we have improved the latter by training them on the meaning and not directly on words.

- Tested and abandoned several types of algorithms and classifiers, because they did not meet our expectations. Therefore, we adopted and adapted the model of recurrent neural networks (LSTM).

From the current state of progress of the project, it can be said that only part of the problems described above has been treated and solved, the other part constitutes the perspectives for this work. We can recall some of them (those which seem strategic and/or important to us): 
- Blockchain technology is very recent, we tried a very first exploitation, deepening its study in order to integrate it more in our processes is a major strategic axis of the perspectives of this work.

- Also, the integration of Blockchain and artificial intelligence seems promising, we have also tried a very first integration of these two technologies of the future. Deepening this study is also another major strategic axis of the perspectives of this work.

- Deepen the study of the characteristics: irony, humour (depending on the culture), metaphor, expressions, etc.; as well as their integration into the process of sentiments analysis and prediction.

- Consider multi-domain and multi-language aspects in sentiments analysis process.

- Implement mechanisms preventing the re-identification of people according to their opinion, comments, etc. many scandals where people have been re-identified from their comments. This question poses an even wider problem on the re-identification of people in Big Data. This is another more important issue of our research.

- Consider nuances of subjectivity which can be expressed through different concepts (Affect, feeling, emotion, and opinion). The distinction between these different aspects and the adaptation of treatments for each constitutes another axis of the perspectives of this work.

- Another perspective concerns the extension of sentiments analysis to other types of data, such as audio and video (multimodal sentiment analysis).

- The integration of the approach in the context of Big Data and its constraints. We have optimized part of the treatments and have dealt with the "unstructured data" and "unavailability/lack" aspects of the processed data, characterizing Big Data, but without considering all its other constraints: acquisition, storage, parallel processing, etc. Taking on all the constraints of Big Data is another perspective of our work.

- Finally, development of a unified system implementing the global "workflow". As explained above, the testing process was done using separate modules and platforms. We therefore envisage the development of other IT modules dedicated to specific tasks, which can be integrated into a single standalone application implementing the entire "Workflow" data processing.

\section{REFERENCES}

Allcott, H., \& Gentzkow, M. (2017). Social media and fake news in the 2016 election. Journal of Economic Perspectives, 31(2), 211-36.

Allcott, H., \& Gentzkow, M. (2017). Social media and fake news in the 2016 election. Journal of Economic Perspectives, 31(2), 211-36.

Azaria, A., Ekblaw, A., Vieira, T., \& Lippman, A. (2016, August). Medrec: Using blockchain for medical data access and permission management. In Open and Big Data (OBD), International Conference on (pp. 25-30). IEEE.

Balmas, M. (2014). When fake news becomes real: Combined exposure to multiple news sources and political attitudes of inefficacy, alienation, and cynicism. Communication Research, 41(3), 430454.

Cachin, C. (2016, July). Architecture of the hyperledger blockchain fabric. In Workshop on Distributed Cryptocurrencies and Consensus Ledgers (Vol. 310).

Del Vicario, M., Quattrociocchi, W., Scala, A., \& Zollo, F. (2018). Polarization and fake news: Early warning of potential misinformation targets. arXiv preprint arXiv:1802.01400.

Esuli, A., \& Sebastiani, F. (2007). SentiWordNet: a high-coverage lexical resource for opinion mining. Evaluation, 17, 1-26.

Frijda, N.H., Mesquita, B., Sonnemans, J., \& Van Goozen, S. (1991). The duration of affective phenomena or emotions, sentiments and passions. 
Ghiassi, M., Skinner, J., \& Zimbra, D. (2013). Twitter brand sentiment analysis: A hybrid system using ngram analysis and dynamic artificial neural network. Expert Systems With Applications, 40(16), 6266-6282.

Juang, C.F., Ku, S.J., \& Huang, H.J. (2004, October). Fuzzy temporal sequence processing by recurrent neural fuzzy network. In Systems, Man and Cybernetics, 2004 IEEE International Conference on (Vol. 6, pp. 5847-5851). IEEE.

Lazer, D.M., Baum, M.A., Benkler, Y., Berinsky, A.J., Greenhill, K.M., Menczer, F., ... Schudson, M. (2018). The science of fake news. Science, 359(6380), 1094-1096.

Marchi, R. (2012). With Facebook, blogs, and fake news, teens reject journalistic "objectivity". Journal of Communication Inquiry, 36(3), 246-262.

Miller, G.A. (1995). WordNet: a lexical database for English. Communications of the ACM, 38(11), 3941.

Mozer, M.C. (1993, February). Neural net architectures for temporal sequence processing. In Santa Fe Institute Studies in The Sciences of Complexity-Proceedings (Vol. 15, pp. 243-243). AddisonWesley Publishing Co.

Pérez-Rosas, V., Kleinberg, B., Lefevre, A., \& Mihalcea, R. (2017). Automatic detection of fake news. arXiv preprint arXiv: 1708.07104.

Pilkington, M. (2016). 11 Blockchain technology: principles and applications. Research Handbook on Digital Transformations, 225.

Rubin, V.L., Conroy, N.J., \& Chen, Y. (2015, January). Towards news verification: Deception detection methods for news discourse. In Hawaii International Conference on System Sciences.

Shand, A.F. (1920). The foundations of character: Being a study of the tendencies of the emotions and sentiments. Macmillan and Company, limited.

Shao, C., Ciampaglia, G.L., Varol, O., Flammini, A., \& Menczer, F. (2017). The spread of fake news by social bots. arXiv preprint arXiv:1707.07592, 96-104.

Shu, K., Sliva, A., Wang, S., Tang, J., \& Liu, H. (2017). Fake news detection on social media: A data mining perspective. ACM SIGKDD Explorations Newsletter, 19(1), 22-36.

Sruk, V., \& Gole, P.A. (2017). Belgrade Faculty of Management, Metropolitan University (p.40). Retrieved from www.icsd.metropolitan.ac.rs

Swan, M. (2015). Blockchain: Blueprint for a new economy. O'Reilly Media, Inc.

Tandoc, E.C., Jr., Lim, Z.W., \& Ling, R. (2018). Defining "fake news" A typology of scholarly definitions. Digital Journalism, 6(2), 137-153.

Teo, K.K., Wang, L., \& Lin, Z. (2000, November 18-21). Wavelet multi-layer perceptron neural network for time-series prediction. In Proc. 10th International Conference on Computing and Information (ICCI 2000, Kuwait).

Wörgötter, F., \& Porr, B. (2005). Temporal sequence learning, prediction, and control: A review of different models and their relation to biological mechanisms. Neural Computation, 17(2), 245319.

Yang, Y., Zheng, L., Zhang, J., Cui, Q., Li, Z., \& Yu, P.S. (2018). TI-CNN: Convolutional neural networks for fake news detection. arXiv preprint arXiv: 1806.00749.

Zhou, X., Tao, X., Yong, J., \& Yang, Z. (2013, June). Sentiment analysis on tweets for social events. In Computer Supported Cooperative Work in Design (CSCWD), 2013 IEEE 17th International Conference on (pp. 557-562). IEEE.

Zhu, Q., Chen, J., Zhu, L., Duan, X., \& Liu, Y. (2018). Wind Speed Prediction with Spatio-Temporal Correlation: A Deep Learning Approach. Energies, 11(4), 705.

Zyskind, G., \& Nathan, O. (2015, May). Decentralizing privacy: Using blockchain to protect personal data. In Security and Privacy Workshops (SPW), 2015 IEEE (pp. 180-184). IEEE. 